American Journal of Infectious Diseases 8 (1): 19-25, 2012

ISSN 1553-6203

(C) 2012 Science Publications

\title{
Molecular Medicine-Prospects and Challenges
}

\author{
${ }^{1}$ Ramchand, C.N., ${ }^{1}$ R. Sripathy, ${ }^{1}$ N. Somashekara, \\ ${ }^{2}$ A. Buch, ${ }^{2}$ H. Pant, ${ }^{2}$ A. Vyas and ${ }^{2}$ R. Upadhyay \\ ${ }^{1}$ Research and Development, Laila Pharmaceuticals Pvt. Ltd., Chennai, India \\ ${ }^{2}$ PD Patel Insitute of Applied Sciences, \\ Charotar University of Science and Technology, Changa, India
}

\begin{abstract}
Drug discovery and development is a process that rationally leads to instigation of compound or formulation for the treatment of particular disease/diseased condition by acting on its specific target (s). Most of the drugs identified by conventional drug discovery process were as a result of unsystematic, random and serendipitous approach. Enhancement of knowledge in the field of biotechnology, molecular biology and medicinal chemistry improved the basic understanding of disease mechanism, mechanism of drug action resulting in a paradigm shift in drug discovery approach towards development of targeted drug discovery. Currently, molecular medicine encompassing elucidation of the genetic basis of disease, diagnosis of the disease and the design of an appropriate approach to disease management or therapy, promises to be an effective strategy for modern drug discovery. The change in approach resulted in improvement in target innovation, however doesn't impact the rate of NMEs approved. The challenges limiting the drug discovery and prospects offered by molecular medicine and reverse pharmacology for its improvement were discussed in this review.
\end{abstract}

Key words: Target innovation, molecular medicine, drug discovery, specific target, conventional drug, serendipitous approach, molecular biology, medicinal chemistry, disease mechanism, discovery approach

\section{INTRODUCTION}

From time immemorial, scientists have been trying to develop therapeutic solutions for alleviating or preventing a variety of diseases that had existed or still exists. In order to develop a therapeutic molecule against any disease, it is very essential to understand the precise mechanism of the disease and the specific target that is associated with it. However, achieving this is a daunting task. Most of the drugs identified by conventional drug discovery process were as a result of unsystematic, random and serendipitous approach. The history of drug discovery shall be considered from the early 19th century, where botanicals were used for therapeutic purpose under the cultural and religious influences. Biologically active organic molecule isolation and its therapeutic application was realized and instigated during the late 19th century (Tsinopoulos and McCarty, 2002). For instance, salicyclic acid, the precursor of aspirin, was isolated in 1874 from willow bark. Various more potent painkillers, such as morphine and codeine, were isolated from the opium poppy, around the same period. Though the mode of drug action and mechanism of disease progression was not well understood, alleviation of symptoms was marked as the accomplishment of that therapeutics.

Discovery of 'microbial theory of disease' to explain pathogenesis of infectious diseases and accidental invention of penicillin led to the era of antibiotics in the early 21 st century. This was the era where discovery of specific targeted drugs emerged shifting from the serendipitous fashion (Tsinopoulos and McCarty, 2002). Enhancement of knowledge in the field of biotechnology, molecular biology and medicinal chemistry improved the basic understanding of disease mechanism, mechanism of drug action and hence a paradigm shift in drug discovery approach towards development of targeted drug discovery was observed. However, the challenge that confronts is in the field of drug discovery is still tremendously complex and unbelievably strenuous. The challenge in the targeted drug discovery and elucidation of disease mechanism still exist resulting in unmet or poorly addressed therapeutic solutions for many diseases such as cancer, Alzheimer's disease and HIV/AIDS. The development of drug resistance and recall of drugs from the market because of its defective or potentially harmful properties intensifies the challenge associated with current drug discovery and development.

Corresponding Author: Ramchand, C.N., Research and Development, Laila Pharmaceuticals Pvt. Ltd., Chennai, India 
Most diseases are complex and multifactorial, resulting from a complex series of events involving changes in the level of expression of many genes and/or environmental factors and behavior. Intervention of one or few of these targets might affect the equilibrium of the cellular events. Identification and monitoring of these changes during disease progression is essential for novel drug target identification and discovery of unique molecules for these targets. According to FDA, the rate of first against target drugs in the last 20 years was found to be constantly 5.3 new drugged targets/year. Currently promising strategy evolving to improve this number is molecular medicine. Molecular medicine encompasses the elucidation of the genetic basis of disease, diagnosis of the disease and the design of an appropriate approach to disease management or therapy (Kresina, 2001). Rapid development in the genomics, proteomics and metabolomics coupled with systems biology and combinatorial chemistry has contributed to the evolution of new dimension of drug discovery called molecular medicine. These strive for identification of novel targets and improve pharmacophores by expanding the knowledge on disease mechanism. This will allow researchers, physician and scientists to use this knowledge in the design of specific molecular tools for disease diagnosis, treatment, prognosis and prevention. This review consolidates the development, prospects and challenges of molecular medicine in drug discovery process.

Paradigm shift in drug discovery process: Drug discovery and development is a process that rationally leads to instigation of compound or formulation for the treatment of particular disease/diseased condition by acting on its specific target (s). This is an intricate process involving colossal amount of time and capital investment. Classical drug discovery is a target, function or physiology based approach. The discovery of such therapeutic molecules was absolutely through serendipity. Both classical and modern drug discovery processes aspire in the development of therapeutic solution for improvement of specific diseased state. In addition to this, modern drug discovery process as opposed to conventional drug discovery process endeavors to improve the success rates and reduce the time and cost involved in drug discovery. Rapid changes and advancement in the field of molecular biology, systems biology and medicinal chemistry have contributed to a better understanding of disease progression, pathogenesis and have redefined the early drug discovery process by significantly reducing the lead identification cost and time. While appreciating the improvement in knowledge and understanding implicated with modern drug discovery, it's imperative to consider the impact of modern drug discovery approaches in the rate of new drug development.

Current status: Over 30,000 human diseases have been identified till date including infections, inflammation, immune dysfunction, CNS dysfunction, mental disorders, gastro intestinal problem, liver/kidney diseases, allergy and asthma, cancer and memory dysfunction. Out of these, substantial understanding of associated disease mechanism was arrived at only for about 5,000 diseases. Target based therapy for these diseases are very sparse and are available only for few diseases. There are over 25,000 diseases for which the basic understanding of the associated mechanism of disease progression still remains a challenge. For instance, most neurological disorders and cancer disease mechanisms are still a mystery to be resolved. Change in expression and activity of thousands of proteins and genes, lack of ideal study model and insufficient knowledge associated with the disease are major hindering factors for development of suitable therapeutic solutions for these diseases. Till today the most prevalent way of treating a disease has been by the use of small molecules which could go and bind to dysfunctional proteins or could inhibit an enzymatic process, worked through agonizing or antagonizing a receptor protein or controlling the ion flow through the cell membrane ionic channel, inhibiting the transport of small molecule. Currently over 200 therapeutic targets that represents several hundred diseases are known and the methods for synthesizing the small molecules through combinatorial synthesis and identifying by high throughput screening has been progressing rapidly. Still the number of molecules entering the clinical trial phase is trifling and declining rapidly.

Analysis of US FDA's Orange Book (for primarily small-molecule drugs) and the Center for Biologics Evaluation and Research (CBER) website (for biological drugs) identifies in excess of 21,000 drug products; however exclusion of duplicate active ingredients, salt forms, supplements, vitamins, imaging agents and so on reduces the number to only 1,357 unique drugs, of which 1,204 are 'small molecule drugs' and 166 are 'biological' drugs. Of the 1,204 small-molecule drugs, 803 are administered orally, 421 parenterals and 275 topical agents (for this analysis, buccal, rectal, inhalational and other such routes of administration are considered as topical agents). A significant number of at least $192(16 \%)$-of the smallmolecule drugs are prod rugs (Overington et al., 2006). The diversity of the small molecule drug targets is still limited. Figure 1 represents the year wise rate of target innovation. The rate of first against target drugs in the last 20 years was found to be constantly 5.3 new drugged targets/year. 


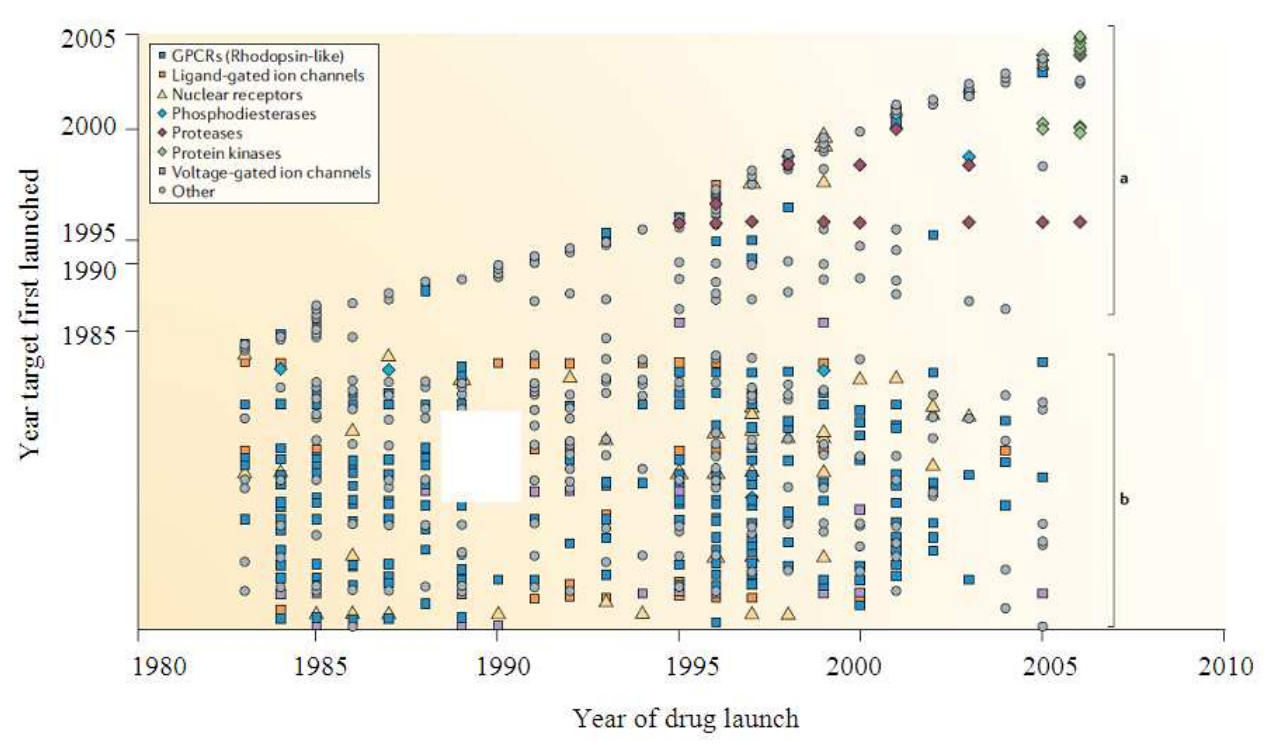

Fig. 1: Rate of Target Innovation. Region a reflects periods of high target innovation. Region b reuse of established mechanisms (Adopted from Overington et al., 2006)

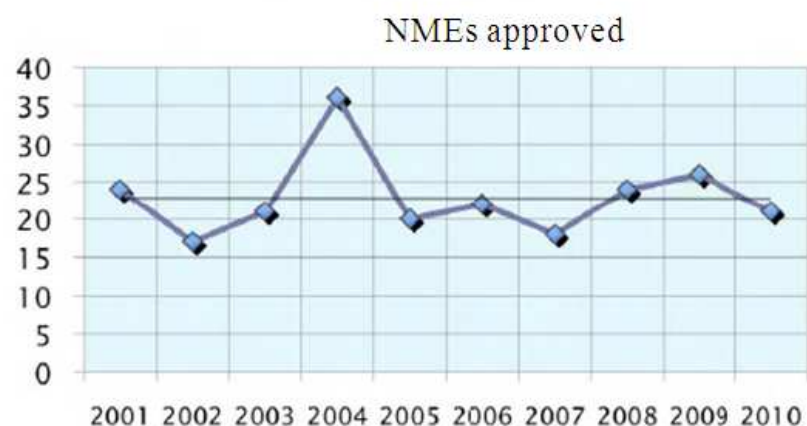

\begin{tabular}{|c|c|}
\hline $\begin{array}{c}\text { Calendar } \\
\text { Year }\end{array}$ & $\begin{array}{c}\text { NMEs } \\
\text { Approved }\end{array}$ \\
\hline 2001 & 24 \\
\hline 2002 & 17 \\
\hline 2003 & 21 \\
\hline 2004 & 36 \\
\hline 2005 & 20 \\
\hline 2006 & 22 \\
\hline 2007 & 18 \\
\hline 2008 & 24 \\
\hline 2009 & 26 \\
\hline 2010 & 21 \\
\hline
\end{tabular}

Fig. 2: Number of NMEs approved by FDA in last 10 years

Currently of the 10,000 compounds screened only 250 compounds enter preclinical studies, among 5000 such compounds entering preclinical studies only one reaches the human trials and finally only $20 \%$ of these compounds entering clinical trials reaches the market. The number of such molecules approved as drugs in the last 10 years averages in the range of 22.9 molecules per annum (Fig. 2). The same doesn't apply to unmet therapeutic areas such as neurological disorders and cancer. About 45 NMEs were approved by FDA in 1995, which is almost twice the average NMEs approved in the last decade. This clearly infers the sharp drop in NMEs approved, even with the rapid development of molecular biology and systems biology. Though, changes in the FDA regulations and decrease in approval of similar or generics has been highlighted as a major cause of the decline in NME approval, other factors such as toxicity, cross reactivity, lack of potential to identify novel drug gable targets and inability of the current "laboratory to clinic", drug discovery approach also contributes significantly.

Intricacy in drug development: The cellular system encompassing cell membrane, nucleic acid, proteins and metabolites are continuously in a process of equilibrium and a minor change in any one molecule can deregulate the total functions in the cell. Change in equilibrium results in alteration in expression of several genes and/or proteins within the cell. These changes are, in general, multi-factorial in nature. Until recently, 
identification of mutated molecule/gene was complex and many times it took several decades to identify the dysfunctional proteins such as enzymes, receptors, ion channels, transport protein, transduction proteins. A combined effort of genomics, proteomics, transcriptomics, metabolomics and the use of microarray technologies, SNP analysis, systems biology and expression analysis have significantly improved the identification of dysfunctional proteins or genes that is involved in a disease condition. This in turn has significant impact in the target innovation (Fig. 1) and hence an opportunity for the discovery of new molecules. As described in Fig. 1, the period between 1985 and 2005 witnessed high rate of target innovation, in addition to the reuse of existing targets and mechanisms. Similarly, the spot light on the protein targets have widened and development of drug for alternate targets such as DNA and RNA have been instigated. Though, this period registered high target innovation (Fig. 1), the rate of new drug molecule approval remains almost static with an average of 22.5 molecules in the last 10 years (Fig. 2). This clearly infers that complexity in development of drug are not limited to target innovation, however the limitations and challenges are diverse and changes with each type of drug target.

Therapeutics targeting DNA: Drugs targeting DNA are either DNA intercalators (proflavin), minor groove binders (Distamycin A, Netropsin), alkylating agents (Benzopyrene) or nucleotide analogs (Cytabine, Gemcitibine). These drugs act by altering/regulating the replication/transcription or induce apoptosis. In addition to these rapid progress has been accomplished in last two decades in development of nucleic acid based therapeutic systems such as gene therapy and DNA vaccines. DNA vaccination promises great potential for fighting a variety of diseases. Initial results are promising and some technologies have advanced to clinical trials. DNA can be introduced by viral or bacterial vectors or through uptake of 'naked' or complexed DNA. Yet challenges remain despite decades of research for safe and efficient delivery of genetic materials and molecules targeting DNA. The use of polymeric materials to elicit delivery holds promise as PLGA, chitosan and PEI has shown potential results in pre-clinical and clinical studies. Recently evolved polymers such as POEs, PAMAMs and PBAEs for polymers tailored gene delivery are also found to be encouraging (Nguyen et al., 2008. Bajaj and Desai, 2006). However, are still in its infancy and much research needs to be done to improve the efficiency with which these delivery system work in humans. Development of novel nonviral delivery strategies for DNA must continue to serve as both methods of biological insight and clinically relevant outcomes. Future development of polymeric and other synthetic materials must focus on these to warrant the success of this molecular medicine.

Therapeutics targeting RNA: RNA therapy is a promising therapeutics for the treatment of so called untreatable diseases such as cancer, viral infections, genetic and metabolic disorders. Anisense RNA, small interfering RNA (Si RNA) and micro RNA (miRNA) has been tested extensively for diverse therapeutic application and its efficacy has been well demonstrated in vitro. A growing number of reports have shown that aberrant miRNA expression is a common feature of human diseases including cancer, which has sparked interest in targeting these regulators of gene expression as a means of ameliorating these diseases. Currently there are 132 RNA/Oligonucleotide based products for ten therapeutic areas, which are in the different stages of development from preclinical to Phase III clinical trials. Interfering RNA can be used for the treatment of cancer, viral diseases (HIV, CMV, Influenza), genetic and metabolic disorders (Huang et al., 2008; Lu et al., 2005; Barik, 2010; Schiffelers et al., 2004; Pai et al., 2006; Harper, 2009; Cabarcas et al., 2010). This RNA/Oligonucleotide therapy is a highly effective method for the treatment of so called, untreatable diseases such as cancer, genetic and other metabolic disorders. Though, RNA therapy has wide range of application, there are certain factors that limit its therapeutic application. Common problems associated with RNA therapy are (Juliano et al., 2009):

- Stability-Stability of the oligonucleotide in blood and other biological fluids

- Safety/Cross reactivity-specific action and prevention of cross reactivity of RNA

- $\quad$ Targeted Delivery-Site directed delivery of RNA to the target

- Intracellular release-Release of RNA in to the cytosol and/or nucleus

- Rapid renal clearance

- Embolization

The limiting factor in the wide spread application of RNA therapy in vivo for the treatment of these diseases is its formulation, administration and delivery of these oligonucleotides selectively to the target site without inducing any toxic or safety issues. Research efforts have been taken to identify an effective method to stabilize the therapeutic 
oligonucleotides in biological fluids/environment, improve its targetability and delivery. Poor stability, cross reactivity, problems associated with delivery to the target site and their weak intracellular penetration in the biological medium complicates the therapeutic application of antisense and miRNA.

As a result, liposome formation, polymeric encapsulation, nanoparticle and magnetic nanoparticle tagging have been identified as effective tools for improving its stability and delivery to some extent but still intracellular release and targatability remains as a challenge to be resolved. Use of colloidal drug carriers such as liposomes and nanoparticles for encapsulation were developed to restrain these problems associated with oligonucleotide therapeutics (Urban-Klein et al., 2005). Among these, magnetic nanoparticles are of potential advantage for the targeted delivery of such antisense oligonucleotides (Pan et al., 2007; Dobson, 2006). Though, magnetic nanoparticles are helpful in selective targeting, embolization of these in veins and capillaries restrain its application in RNA therapy. The major problem associated with these magnetic nanoparticles is its aggregation and its dissolution in biological medium. For in vivo uses, this is quite important as aggregation within the vasculature has the potential for problems such as embolization. Though many of these challenges has been addressed, targeted delivery, intracellular release, embolization and rapid renal clearance still remains a major hurdle for the Antisense/mi-RNA therapy. Hence, improving the antisense/micro RNA delivery using novel delivery system will open up a new arena for RNA therapeutics.

Drugs targeting proteins: Most common protein targets for which successful drugs have been developed include proteases, kinases, G Protein Coupled Receptors (GPCRs) and nuclear hormone receptors. GPCRs (23\%) and enzymes (50\%) represent the most important target classes of proteins for drug discovery (Zheng et al., 2006). It's been estimated that there are about 2000-3000 drug gable proteins in humans where majority of it is identified to be proteases, protein kinases and GPCRs (Hopkins and Groom, 2002; Russ and Lampel, 2005). Most of the currently available drugs with a known mode-of-action shall act through 324 distinct molecular drug targets. Of these, 266 are human-genome-derived proteins and the remainders are bacterial, viral, fungal or other pathogenic organism targets. Small molecule drugs modulate 248 proteins, of which 207 are targets encoded by the human genome. Oral small-molecule drugs target 227 molecular targets, of which 186 are human targets (Overington et al., 2006; Zheng et al., 2006; Lauss et al., 2007). Though all these suggest that proteins are the most widely preferred drug target, development of drug for protein is still an intricate and strenuous process. Complexity in development of drugs targeting proteins are cross reactivity, toxicity and development of drug resistance. This is more pronounced in synthetic molecules, whereas the problem is comparatively less in molecules obtained from natural sources for instance molecules from ethanobotanical sources.

Swinney in his review elucidated and discussed the importance of biochemical mechanism of action of NMEs, approved between 2001 and 2004, for optimal efficacy and safe dosing. The successful therapeutic application of the drug depends not only on the efficacious drug action but also ensuring it at a safe and nontoxic dose. Swinney identified three biochemical operations defined the modes of action of these NMEs:

- Mass action competition (equilibrium)

- A drug stabilized conformational change in the target that is important to the response (conformational) and/or

- Drug action is less-responsive to mass action competition with effectors due to non-equilibrium kinetics (non-equilibrium kinetic)

Approximately $80 \%$ of the NMEs elicit a response utilizing conformational and/or non-equilibrium kinetic mechanisms. The remaining $20 \%$ of NMEs find mass action competition with the endogenous substrate or ligand sufficient for therapeutic utility. These observations suggest that for majority of drug targets, mass action driven equilibrium binding alone may not be sufficient for maximal therapeutic utility. The challenge here is to understand the initial binding interaction as well as to understand and optimize component(s) of initial binding such as conformation, kinetic and thermodynamic properties that are essential for coupling the initial binding to the desired therapeutic response. Consideration of this relationship in drug identification and optimization strategies will facilitate the identification of molecules with optimal therapeutic index (Swinney, 2006). According to Swinney, the toxicity of NMEs can be minimized by providing a maximal response at a low dose to minimize off-target toxicities, or by providing a potential mechanism to minimize the incidence of mechanism-based toxicity while retaining a sufficiently efficacious response.

Reverse pharmacology and systems biology: Major all time bottlenecks in drug discovery are cost, time and toxicity. Current 'lab to clinics' strategy witnessed failure off molecules in clinical trials due to toxicity and inappropriate pharmacokinetic properties. 

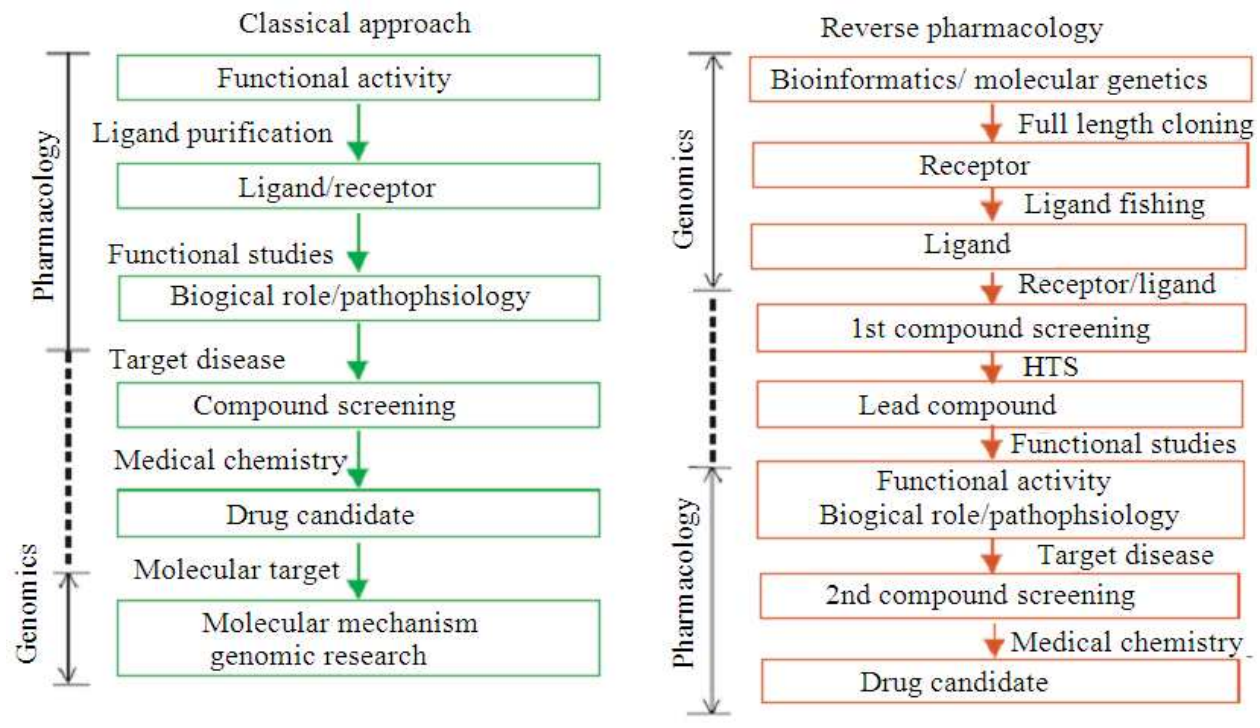

Fig. 3: Comparison of classical and reverse pharmacology approaches in Drug discovery (Adapted from Takenaka, 2001)

A new trans-disciplinary endeavor, with 'clinics to lab' approach called Reverse Pharmacology (RP) has recently emerged and is expected to address these bottlenecks of drug discovery (Fig. 3). RP is an effective strategy, where in scientific evaluation and validation of the traditional ayurvedic, sidhha, unani and other ethanobotanical treatment systems. This comprises of three phases. First the experiential phase comprises clinical observations of biodynamic effects as a result of these traditional medicinal systems. Second, the exploratory studies for establishment of target, mechanism of action, active principle, dose range, tolerability, drug-interactions, dose-range finding in ambulant patients of defined subsets of the disease and paraclinical studies in relevant in vitro and in vivo models to evaluate the target-activity. Third phase includes experimental studies, basic and clinical, at several levels of biological organization, to identify and validate the reverse pharmacological correlates of drug safety and efficacy (Takenaka, 2001; Patwardhan et al., 2008). This approach is valuable in exploring the traditional, nontoxic and safe treatment systems. The scope of reverse pharmacology adds scientific value though elucidation of mechanisms of action at multiple levels of biology and to optimize safety, efficacy and acceptability of the leads in natural products.

Reverse pharmacology offers a major paradigm shift in drug discovery. Failure of molecules in the clinical stage due to poor efficacy, toxicity and poor pharmacokinetic properties can be considerably reduced by RP approach thereby it can significantly reduce the cost and time in drug discovery. In contrast to classical approach in RP systems biology, genomics and proteomics aids the initiation of drug discovery process with elucidation of mechanism drug action and lead identification (Richard and Sriram, 2005). Thus RP in short aims to identify and develop leads from documented clinical and experiential hits thorough interdisciplinary exploratory studies on defined targets in vitro and in vivo.

\section{CONCLUSION}

Drug discovery process has co-evolved with the developments in different fields of medicinal science. The specific targeted drug discovery has been strengthened as evidenced by the discovery of number of novel targets in the past two decades than ever before. Still the rate of NMEs approved remained unchanged, possibly due to the unmet limitations in the molecular medicine. Some of those predominant limitations such as in vivo efficacy, toxicity and safety issues can be addressed by application of systems biology and reverse pharmacology approach. Rapid growth and continuous improvements in the field of molecular medicine also promises to resolve problems associated with poor pharmacokinetics though advent of number of site directed and novel nanotechnology based drug delivery systems. With the advent of new strategies and renewal of the existing science of molecular medicine, improvement in drug development and appliance of novel molecules especially for untreatable diseases shall be expected in decades to come. 


\section{REFERENCES}

Bajaj, A. and M. Desai, 2006. Challenges and strategies in novel drug delivery technologies. Pharma Times, 38: 12-16.

Barik, S., 2010. siRNA for influenza therapy. Viruse, 2: 1448-1457. DOI: $10.3390 / \mathrm{v} 2071448$

Cabarcas S., K. Watabe and L. Schramm, 2010. Inhibition of U6 snRNA transcription by PTEN. OnLine J. Biol. Sci., 10: 114-125. DOI: 10.3844/ojbsci.2010.114.125

Dobson, J., 2006. Gene therapy progress and prospects: magnetic nanoparticle-based gene delivery. Gene Therapy, 13: 283-287. PMID: 16462855

Harper, S.Q., 2009. Progress and challenges in RNA interference therapy for Huntington disease. Arch. Neurol., 66: 933-938. PMID: 19667213

Hopkins, A.L. and C.R. Groom, 2002. The druggable genome. Nat. Rev. Drug Discov., 1: 727-730. PMID: 12209152

Huang, C., M. Li, C. Chen and Q. Yao, 2008. Small interfering RNA therapy in cancer: Mechanism, potential targets and clinical applications. Expert Opin. Ther. Targets, 12: 637-645. PMID: 18410245

Juliano, R., J. Bauman, H. Kang and X. Ming, 2009. Biological Barriers to Therapy with Antisense and siRNA Oligonucleotides. Mol. Pharm., 6: 686-695. PMID: 19397332

Kresina, T.F., 2001. An Introduction to Molecular Medicine and Gene Therapy. 1st Edn., A John Wiley and Sons, Inc., Publication, New York, ISBN-10: 0471391883, pp: 386.

Lauss, M., A. Kriegner, K. Vierlinger and C. Noehammer, 2007. Characterization of the drugged human genome. Pharmacogenomics, 8: 1063-1073. PMID: 17716238

Lu, P.Y., F. Xie and M.C. Woodle, 2005. In vivo application of RNA interference: From functional genomics to Therapeutics. Adv. Genet., 54: 115142. PMID: 16096010

Nguyen, D.N., J.J. Green, J.M. Chan, R. Langer and D.G. Anderson, 2009. Polymeric materials for gene delivery and DNA vaccination. Adv. Mater., 21: 847-867. DOI: $10.1002 /$ adma.200801478

Overington, J.P., B. Al-Lazikani and A.L. Hopkins, 2006. How many drug targets are there? Nature Rev. Drug Discovery, 5: 993-996. DOI: $10.1038 / \mathrm{nrd} 2199$
Pai, S.I., Y.Y. Lin, B. Macaes, A. Meneshian and C.F. Hung et al., 2006. Prospects of RNA interference therapy for cancer. Gene Ther., 13: 464-477. PMID: 16341059

Pan, B., D. Cui, Y. Sheng, C. Ozkan and F. Gao et al., 2007. Dendrimer-modified magnetic nanoparticles enhance efficiency of gene delivery system. Cancer Res., 67: 8156-8163. DOI: 10.1158/00085472.CAN-06-4762

Patwardhan, B., A.D.B. Vaidya, M. Chorghade and S.P. Joshi, 2008. Reverse pharmacology and systems approaches for drug discovery and development. Curr. Bioactive Compounds, 4: 201-212.

Richard, R.J.A. and N. Sriraam, 2005. A feasibility study of challenges and opportunities in computational biology: A Malaysian perspective. Am. J. Applied Sci., 2: 1296-1300. DOI: 10.3844/ajassp.2005.1296.1300

Russ, A.P. and S. Lampel, 2005. The druggable genome: An update. Drug Discov. Today, 10: 1607-1610. PMID: 16376820

Schiffelers, R.M., A. Ansari, J. Xu, Q. Zhou and Q. Tang et al., 2004. Cancer siRNA therapy by tumor selective delivery with ligand-targeted sterically stabilized nanoparticle. Nucleic Acids Res., 32: e149-e149. PMID: 15520458

Swinney, D.C., 2006. Biochemical mechanisms of New Molecular Entities (NMEs) approved by United States FDA during 2001-2004: Mechanisms leading to optimal efficacy and safety. Curr. Topics Med. Chem., 6: 461-478. PMID: 16719803

Takenaka, T., 2001. Classical Vs reverse pharmacology in drug discovery. BJU. Int., 88: 7-10. PMID: 11589663

Tsinopoulos, C. and I. McCarthy, 2002. An evolutionary classification of the strategies for drug discovery. University of Warwick, UK.

Urban-Klein, B., S. Werth, S. Abuharbeid, F. Czubayko and A. Aigner, 2005. RNAi-mediated genetargeting through systemic application of polyethylenimine (PEI)-complexed siRNA in vivo. Gene Ther., 12: 461-466. PMID: 15616603

Zheng, C.J., L.Y. Han, C.W. Yap, Z.L. Ji and Z.W. Cao et al., 2006. Therapeutic targets: Progress of their exploration and investigation of their characteristics. Pharma. Rev., 58: 259-279. DOI: 10.1124/pr.58.2.4 